\title{
Relationship Between Polymorphisms in Methotrexate Pathway Genes and Outcome of Methotrexate Treatment in a Cohort of 119 Patients with Juvenile Idiopathic Arthritis
}

\author{
Mojca Zajc Avramovič, Vita Dolžan, Nataša Toplak, Meta Accetto, Lara Lusa, and Tadej Avčin
}

ABSTRACT. Objective. To identify clinical and pharmacogenetic determinants of efficacy and toxicity of methotrexate (MTX) in juvenile idiopathic arthritis (JIA) over time.

Methods. A cohort of 119 consecutive patients with JIA treated with MTX was reviewed. The Juvenile Arthritis Disease Activity Score including 71 joints was used to measure disease activity. Nonresponders were patients who did not reach a minimum of $30 \%$ improvement after 6 months of treatment or were switched to biologic drugs in the first 6 months because of inefficacy. All adverse events (AE) were noted. Genotyping of single-nucleotide polymorphisms (SNP) in the genes coding for MTX transporters, folate pathway, and adenosine pathway was performed using real-time PCR methods. Univariate and multivariable penalized logistic and Cox regression were used to analyze data.

Results. Thirty patients $(25.8 \%)$ were defined as nonresponders and 55 (47.2\%) were switched to biologics during the followup. Sixty-five patients (54.5\%) reported AE in a total of 405 patient-years, and 10 patients $(8.4 \%)$ discontinued MTX because of AE. AMPD1 rs17602729 and MTHFD1 rs 2236225 were associated with gastrointestinal AE while the latter together with MTRR rs 1801394 also demonstrated associations with developing hepatoxicity. MTHFR rs 1801131, ABCG2 rs2231137, wild-type of MTR rs1805087, and wild-type of $A B C C 2$ rs2273697 were identified as potential markers for discontinuing MTX treatment because of AE. MTHFR rs1801133, MTRR rs 1801394, and ABCC2 rs 2273697 were associated with switching to biologics.

Conclusion. SNP in different MTX metabolic pathways influence treatment with MTX. Genetic variability is a better marker for toxicity than efficacy. (First Release June 1 2017; J Rheumatol 2017;44:1216-23; doi:10.3899/jrheum.160950)

\author{
Key Indexing Terms: \\ JUVENILE IDIOPATHIC ARTHRITIS \\ PHARMACOGENETIC DETERMINANTS \\ DRUG EFFICACY
}

\author{
METHOTREXATE \\ SINGLE-NUCLEOTIDE POLYMORPHISMS \\ DRUG TOXICITY
}

Juvenile idiopathic arthritis (JIA) is one of the most common chronic diseases in childhood, with a prevalence of 1 in 1000 children. Methotrexate (MTX) in a dose of 10-15 $\mathrm{mg} / \mathrm{m}^{2} /$ week is the most important disease-modifying antirheumatic drug (DMARD) used in JIA and is recommended as an initial DMARD therapy ${ }^{1}$. Around 65\%-70\% of children reach and sustain remission with MTX. In the remaining patients, the delay in optimal treatment can lead to functional disability, and therefore it would be of great clinical importance to identify MTX-refractory patients earlier ${ }^{2}$.

While MTX has a long history as a drug, its precise mode of action is still not completely understood. MTX enters the cell by the solute carrier 19A1 and is pumped out of the cell
From the Department of Allergology, Rheumatology and Clinical Immunology, Children's Hospital, University Medical Centre Ljubljana; Institute of Biochemistry, and Department of Pediatrics, and Institute for Biostatistics and Medical Informatics, Medical Faculty, University of Ljubljana, Ljubljana, Slovenia.

Partially supported by the Slovenian Research Agency grants L3-4150, P3-0343, and P1-0170, and by the University Medical Center Ljubljana research grant 20140208.

M. Zajc Avramovič, MD, Department of Allergology, Rheumatology and Clinical Immunology, Children's Hospital, University Medical Centre Ljubljana; V. Dolžan, $M D, P h D$, Institute of Biochemistry, Medical Faculty, University of Ljubljana; $N$. Toplak, MD, PhD, Department of Allergology, Rheumatology and Clinical Immunology, Children's Hospital,

\author{
University Medical Centre Ljubljana, and Department of Pediatrics, \\ Medical faculty, University of Ljubljana; $M$. Accetto, MD, Department of \\ Allergology, Rheumatology and Clinical Immunology, Children's Hospital, \\ University Medical Centre Ljubljana; L. Lusa, PhD, Institute for \\ Biostatistics and Medical Informatics, Medical Faculty, University of \\ Ljubljana; T. Avčin, MD, PhD, Department of Allergology, Rheumatology \\ and Clinical Immunology, Children's Hospital, University Medical Centre \\ Ljubljana, and Department of Pediatrics, Medical faculty, University of \\ Ljubljana. \\ Address correspondence to Dr. T. Avčin, Department of Allergology, \\ Rheumatology and Clinical Immunology, Children's Hospital, Bohoričeva \\ 20, 1000 Ljubljana, Slovenia.E-mail:tadej.avcin@kclj.si \\ Accepted for publication April 5, 2017.
}


by ATP-binding cassette (ABC) transporters. Inside the cell, activated MTX polyglutamates inhibit several enzymes in the folate pathway, thereby disrupting the purine and pyrimidine synthesis. It is generally assumed that MTX in the dose used for rheumatic diseases does not considerably inhibit cell division $^{3,4}$. MTX also inhibits the enzymes in the adenosine pathway, resulting in the release of endogenous adenosine, which is thought to be responsible for the site-specific antiinflammatory effects of MTX ${ }^{3}$.

There is growing evidence that single-nucleotide polymorphisms (SNP) within the MTX pathway genes are significant contributors to interindividual differences in response to MTX and adverse events (AE) in adults ${ }^{3,5,6,7}$, as well as patients with JIA ${ }^{8-17}$. However, several candidate SNP that emerged from other fields of medicine were not evaluated in JIA $^{18,19}$. To our knowledge, no study included SNP in all known MTX metabolic pathways and assessed toxicity and efficacy over time in JIA. Therefore, the aim of our study was to identify clinical and pharmacogenetic factors to predict efficacy and toxicity of MTX in JIA over time.

\section{MATERIALS AND METHODS}

Study design and patient selection. The design of our study was a single-center, descriptive observational cohort study. The data were collected retrospectively with a longitudinal followup. The inclusion criteria were a definite diagnosis of JIA according to the International League of Associations for Rheumatology criteria ${ }^{20}$ and past or current treatment with MTX. In total, 126 consecutive patients with JIA treated with MTX at the University Children's Hospital Ljubljana from September 2011 to October 2014 were eligible to participate in the study. The study was approved by the National Ethics Committee for Research in Medicine and was carried out according to the Helsinki Declaration (approval number 39/11/09). Patients with all JIA subtypes were recruited at their regular visits. Seven patients were excluded from the study because they refused to participate ( $\mathrm{n}$ $=2)$, received MTX for isolated uveitis $(n=4)$, or had missing data $(n=1)$. All patients were Central European white and their parents or guardians gave their written informed consent prior to enrollment.

Data were retrospectively collected from the medical records for 78/119 patients $(65 \%)$ who were already taking MTX at the start of the study or had been taking MTX in the past. Forty-one patients (34\%) started taking MTX during the course of our study and their data were collected prospectively. Patients were followed up at regular intervals by the standardized protocol used at our department until the study was finished. Patients were evaluated at least 4 times: upon MTX introduction, 3 months and 6 months after treatment was introduced, and at the last followup visit. All patients were also evaluated in case of a flare. Starting oral dose of MTX was $10 \mathrm{mg} / \mathrm{m}^{2}$ and increased to $15 \mathrm{mg} / \mathrm{m}^{2}$ in the first 3 months if required.

Concomitant treatment with nonsteroidal antiinflammatory drugs, bridging therapy with oral corticosteroids, and intraarticular corticosteroid injections were allowed. All patients were receiving folic acid supplementation. Biologic therapy because of refractory disease was introduced by a standard protocol used in our department.

Definition of response. Response to treatment was measured using the Juvenile Arthritis Disease Activity Score including 71 joints (JADAS-71) ${ }^{21}$. The response was measured as a percentage of improvement in the JADAS-71 score at 3 and 6 months after treatment introduction and at the last followup visit. Nonresponders were patients who did not achieve the minimum improvement of 30\% in the score 6 months from baseline and those who required therapy escalation to biologic drugs because of inefficacy in the first 6 months.
Inactive disease, remission while taking or not taking therapy, and flares were noted. Inactive disease was defined as JADAS score of a maximum of $1^{22}$, remission while taking therapy as continuously inactive disease for 6 months, and remission while not taking therapy as continuously inactive disease for 12 months without any antiarthritis drug ${ }^{23}$. Flare was defined as worsening of the disease that required treatment intervention after inactive disease had already been achieved.

Recording of AE. All AE that were reported by the patients throughout the course of MTX treatment were recorded. AE were grouped by organ systems: (1) gastrointestinal $\mathrm{AE}$ (GAE): nausea, vomiting, abdominal pain; (2) hepatotoxicity: serum transaminases above the upper range of normal; (3) bone marrow toxicity; (4) dermatological complaints; (5) central nervous system toxicity; (6) renal toxicity; (7) infections; and (8) allergic reactions. The AE were divided into categories of mild, moderate, and severe in accordance with the Common Terminology Criteria for $\mathrm{AE}^{24}$. All AE that were the reason for the patient to discontinue the treatment were noted separately. Genotyping. In total, $5 \mathrm{ml}$ of peripheral blood was collected into tubes with sodium citrate and stored for DNA isolation. Genomic DNA was isolated from peripheral blood leukocytes using commercial kits (Flexigene, Qiagen). SNP were analyzed with real-time PCR-based methods using TaqMan (Applied Biosystems) and KASPar assays (KBiosciences). The following polymorphisms were determined in folate pathway: MTHFD1 rs2236225, MTHFR rs1801133, MTHFR rs1801131, MTR rs1805087, and MTRR rs1801394; adenosine pathway: ATIC rs2372536, AMPD1 rs17602729, and ITPA rs 1127354; and in the genes for MTX transporters: $A B C C 2$ rs2804402, $A B C G 2$ rs 2231142, $A B C C 2$ rs2273697, $A B C B 1$ rs 1045642, $A B C G 2$ rs2231137, SLC19A1 rs1051266, ABCC2 rs717620, SLCO1B1 rs11045879, SLCO1B1 rs4149056, and SLCO1B1 rs2306283. Polymorphic TYMS region was analyzed by multiplying promoter region as previously described ${ }^{25}$. PCR products were analyzed on $3 \%$ agarose gel, and visualized using ethidium bromide staining.

Statistical analysis. Patient characteristics of responders and nonresponders were compared using the 2-tailed Fisher's exact test for categorical data, and the 2 sample Student $t$ test was used for numerical data. Log-rank test was used to compare responders and nonresponders in time-to-event endpoints (time to inactive disease, remission, $\mathrm{AE}$, escalation to biological treatment). Univariate logistic regression models were used to estimate the association between JADAS 30\% nonresponse after 6 months and each of the polymorphisms. Results were reported as OR with $95 \% \mathrm{CI}$ and $\mathrm{p}$ values. A significance level of $\alpha=0.05$ was used in the univariate analyses. We also estimated a multivariable logistic regression model with the Least Absolute Shrinkage and Selection Operator (LASSO) penalty, which included all the polymorphisms; the use of this model is appropriate when the number of covariates is large compared with the number of events, and it performs some type of variable selection: the polymorphisms whose penalized OR were not shrunk to 1 were considered as associated to MTX response. The dominant genetic model was used. Wild-type was the reference category.

A similar approach was used for time-to-event endpoints: univariable and multivariable cause-specific Cox models with LASSO penalization were used to estimate the association between the polymorphisms and a specific event; the univariable results were summarized with $\mathrm{HR}, 95 \% \mathrm{CI}$, and $\mathrm{p}$ values, the multivariable with $\mathrm{HR}$. All statistical analyses were carried out using the $\mathrm{R}$ language ${ }^{26}$.

\section{RESULTS}

Patient characteristics. A total of 119 patients were included in the analysis, 91 girls ( $76.5 \%$ ) and 28 boys (23.5\%). Patient characteristics are presented in Table 1. Mean time of disease duration before MTX was started was 13 months in the whole cohort and 6.9 months in the subgroup of patients with polyarticular disease (rheumatoid factor-positive and -negative). Mean starting dose of MTX was $10.2 \mathrm{mg} / \mathrm{m}^{2}$ and

Personal non-commercial use only. The Journal of Rheumatology Copyright (c) 2017. All rights reserved. 
Table 1. Patient group characteristics and characteristics of responders and nonresponders. Values are mean (SD) unless otherwise specified.

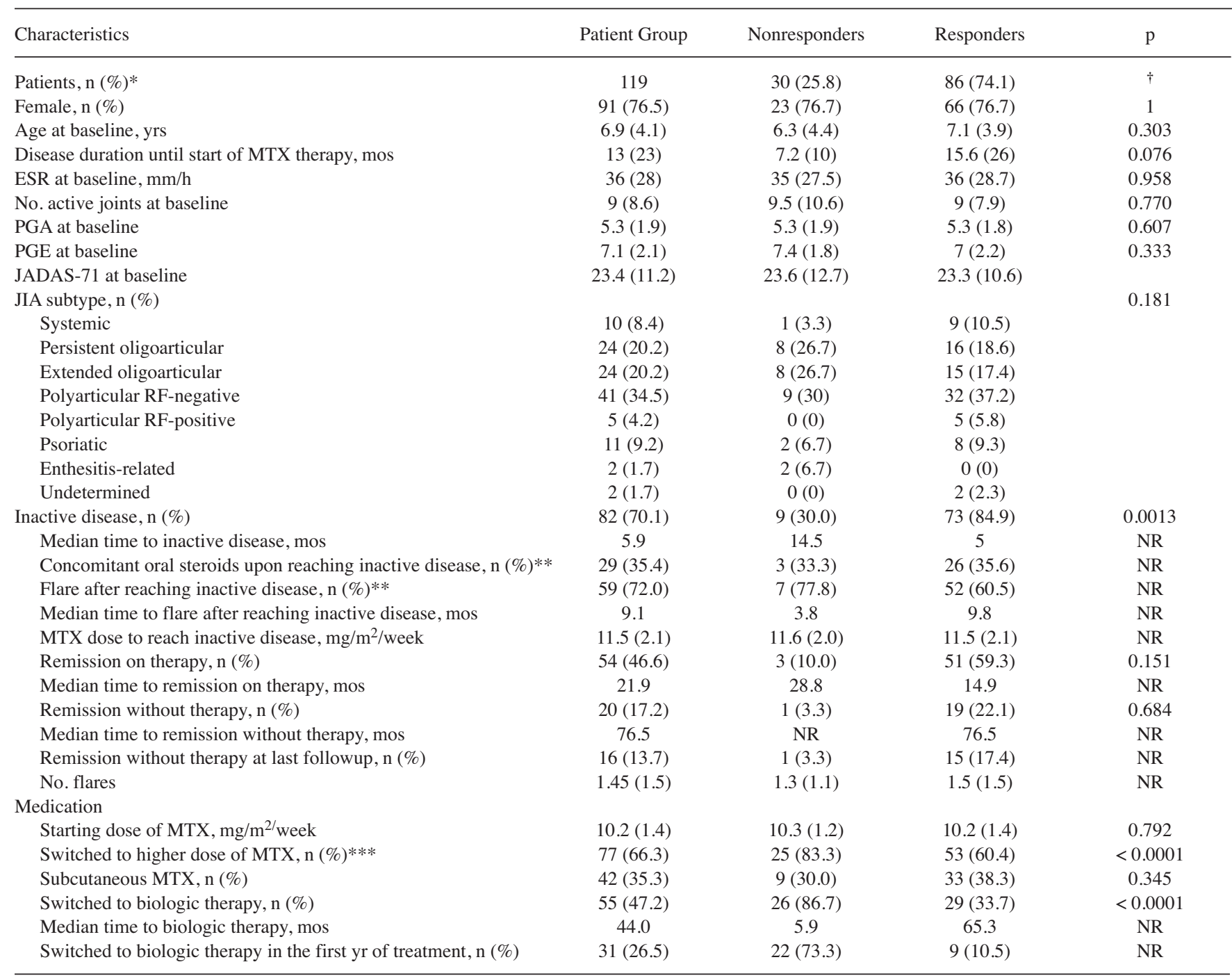

* Three patients were excluded from the efficacy study. ** Analyses performed only in the patients who reached inactive disease. *** Higher dose is $>12.5$ $\mathrm{mg} / \mathrm{m}^{2} /$ week. ${ }^{\dagger} \mathrm{p}$ value comparing responders and nonresponders. ESR: erythrocyte sedimentation rate; PGA: physician's global assessment; PGE: parent's general evaluation of well-being; JADAS-71: Juvenile Arthritis Disease Activity Score including 71 joints; JIA: juvenile idiopathic arthritis; RF: rheumatoid factor; MTX: methotrexate; NR: not reported.

mean dose at 6 months was $12.0 \mathrm{mg} / \mathrm{m}^{2}$. Twenty patients $(16.8 \%)$ discontinued MTX in less than 6 months because of inefficacy or toxicity. At 6 months, $48.0 \%$ of the patients still taking MTX were receiving a higher dose. Forty-two patients (35\%) were switched to subcutaneous MTX to achieve higher efficacy. The mean followup of our patients from the beginning of the disease was 45 months.

Treatment efficacy. Out of 116 patients evaluated for efficacy, $30(25.8 \%)$ were defined as MTX nonresponders at 6 months of therapy. The following patients were excluded from efficacy assessment: 1 because of missing data, 1 because of MTX withdrawal before reaching 6 months of treatment following severe $\mathrm{AE}$, and 1 because of changed indication for treatment. The groups of MTX responders and nonre- sponders did not differ statistically in any of the clinical variables evaluated at baseline (Table 1). Disease duration prior to treatment was not associated to efficacy of MTX $(\mathrm{p}>0.05)$.

Analysis of AE. AE data are presented in Table 2. Sixty-five patients $(54.5 \%)$ reported $\mathrm{AE}$ in a total of 405 patient-years. Most patients with AE (83\%) developed them in the first year of treatment. The most common AE were gastrointestinal, which occurred in 39 patients (32.8\%), followed by hepatotoxicity in 28 patients $(23.5 \%)$. In addition, 25 patients $(21.0 \%)$ had moderate hepatotoxicity and were included in the further analyses. AE were considered mild in $50(42 \%)$ and moderate in 43 patients $(36.1 \%)$. A total of 36 patients $(30.3 \%)$ reported infectious illness during the study period; 
Table 2. Adverse events in patient population.

\begin{tabular}{lc}
\hline Adverse Events & $\mathrm{n}(\%)$ \\
\hline Overall adverse events & $65(54.5)$ \\
$\quad$ Estimated cumulative incidence after 6 mos/12 mos* & $40.0 \% / 51.2 \%$ \\
Severe AE & $14(11.8)$ \\
Discontinued MTX because of AE & $10(8.4)$ \\
Discontinued MTX because of AE while receiving & \\
$\quad$ MTX monotherapy & $7(5.9)$ \\
$\quad$ Estimated cumulative incidence after 6 mos/12 mos* & $3.1 \% / 5.8 \%$ \\
Gastrointestinal AE & $39(32.8)$ \\
$\quad$ Estimated cumulative incidence after 6 mos/12 mos* & $21.5 \% / 31.9 \%$ \\
Hepatotoxicity & $28(23.5)$ \\
Moderate hepatotoxicity & $25(21.0)$ \\
$\quad$ Estimated cumulative incidence after 6 mos/12 mos* & $13.5 \% / 16.3 \%$ \\
Infections & $36(30.3)$ \\
Severe infections & $13(10.2)$ \\
Dermatological AE & $11(9.2)$ \\
Hematological AE & $1(0.8)$ \\
Renal AE & $0(0)$ \\
Central nervous system AE & $1(0.8)$ \\
\hline
\end{tabular}

* Estimated proportion of patients who experienced the event in the first 6 months/12 months of therapy. Estimates were obtained using Kaplan-Meier method. AE: adverse events; MTX: methotrexate.

however, only 13 patients (10.9\%) reporting severe infection were included in further analyses. One patient had a severe $\mathrm{AE}$ of persistent leukopenia. Apart from these, no other severe $\mathrm{AE}$ were noted.

Of the 10 patients $(8.4 \%)$ who discontinued MTX because of AE, 4 had nausea/vomiting, 4 had persistently elevated liver transaminases, 1 had persistent leukopenia, and 1 discontinued MTX because of worsening of an existing dermatological condition. Three patients developed AE after biologic therapy was introduced and were not included in further analyses. We estimated that $3.1 \%$ of patients discon- tinued MTX because of AE in the first 6 months and $5.8 \%$ in 1 year.

Pharmacogenetic determinants of MTX treatment efficacy. All of the investigated SNP except $T S^{*} 2 / * 3$ were in Hardy-Weinberg equilibrium. The minor allele frequencies and genotype frequencies are shown in Supplementary Table 1 (available with the online version of this article). The number of successful analyses per each SNP and numbers of polymorphic alleles are shown in Supplementary Table 2 (available with the online version of this article). Significant results are shown in Table 3 and Table 4, and all results are shown in Supplementary Table 3 (available with the online version of this article). JADAS 30\% nonresponse after 6 months was associated with $A B C C 2$ rs 2804402 genotype $(\mathrm{p}=0.048)$ in univariate analysis, but the penalized multivariable logistic regression analysis did not confirm the association. However, this SNP, together with 5 others, was statistically significantly associated with time to reach inactive disease in multivariable penalized Cox regression [folate pathway: MTR rs1805087 (HR 1.58), MTHFR rs1801131 (HR 1.19); adenosine pathway: ITPA rs1127354 (HR 1.36), ATIC rs2372536 (HR 1.14); MTX transporters: $A B C C 2$ rs2273697 (HR 1.55 for wild-type), and $A B C C 2$ rs2804402 (HR 1.07)]. No associations were found between the investigated SNP and time to flare or reaching remission while taking and not taking therapy (Supplementary Table 3, available with the online version of this article).

Pharmacogenetic determinants of MTX AE. Statistically significant results are shown in Table 5 and Table 6, and all results are shown in Supplementary Table 4 (available with the online version of this article). These were associated to time to develop GAE in the univariate analyses: AMPDI rs17602729 ( $\mathrm{p}=0.011)$ from adenosine pathway, MTHFD1 rs2236225 ( $\mathrm{p}=0.015)$ from folate pathway, and MTX trans-

Table 3. Pharmacogenetic determinants of MTX treatment efficacy. Results of all performed analyses are shown in Supplementary Table 3 (available with the online version of this article).

\begin{tabular}{|c|c|c|c|c|c|c|}
\hline \multirow[t]{4}{*}{ SNP } & \multicolumn{3}{|c|}{ Nonresponse to MTX } & \multicolumn{3}{|c|}{ Time to Inactive Disease } \\
\hline & \multirow[b]{3}{*}{ Associated Allele, OR, 95\% CI } & & \multirow{3}{*}{$\begin{array}{l}\text { Penalized Cox } \\
\text { Regression OR }\end{array}$} & \multirow{2}{*}{\multicolumn{2}{|c|}{ Univariate Analysis }} & \multirow{3}{*}{$\begin{array}{r}\text { Penalized Cox } \\
\text { Regression, HR }\end{array}$} \\
\hline & & & & & & \\
\hline & & $\mathrm{p}$ & & Associated Allele, HR, 95\% CI & $\mathrm{p}$ & \\
\hline \multicolumn{7}{|l|}{ Folate pathway } \\
\hline MTHFR rs 1801133 & $\mathrm{~T}, 0.64,0.27-1.49$ & 0.299 & 1 & $\mathrm{~T}, 1,0.64-1.56$ & 0.999 & 1 \\
\hline MTHFR rs 1801131 & $C, 0.66,0.28-1.52$ & 0.325 & 1 & C, $1.26,0.8-1.97$ & 0.317 & 1.19 \\
\hline \multicolumn{7}{|l|}{ Adenosine pathway } \\
\hline ATIC $\mathrm{rs} 2372536$ & $\mathrm{G}, 0.99,0.43-2.33$ & 0.984 & 1 & G $1.18,0.76-1.85$ & 0.457 & 1.14 \\
\hline ITPA rs 1127354 & A, $1.57,0.42-5.17$ & 0.480 & 1 & $\mathrm{~A}, 1.6,0.82-3.11$ & 0.170 & 1.36 \\
\hline \multicolumn{7}{|l|}{ MTX transporters } \\
\hline$A B C C 2$ rs 2804402 & G, 0.37, 0.14-0.99 & 0.048 & 1 & $\mathrm{G}, 1.14,0.65-2.01$ & 0.640 & 1.07 \\
\hline$A B C C 2$ rs 2273697 & A, $1.06,0.45-2.44$ & 0.894 & 1 & G wt, $0.62,0.39-0.99$ & 0.043 & 1.55 \\
\hline
\end{tabular}

Wt was associated with tested variable, and in all other analyses, polymorphic allele was associated with tested variable. Significant data are in bold face. MTX: methotrexate; SNP: single-nucleotide polymorphism; wt: wild-type.

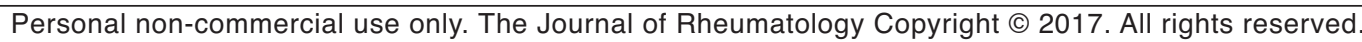


Table 4. Pharmacogenetic determinants of escalation to biologic drugs because of inefficacy or AE. Results of all performed analyses are shown in Supplementary Table 3 (available with the online version of this article).

\begin{tabular}{|c|c|c|c|}
\hline \multirow[t]{2}{*}{ SNP } & \multicolumn{2}{|l|}{ Univariate Analysis } & \multirow{2}{*}{$\begin{array}{c}\text { Penalized } \\
\text { Cox } \\
\text { Regression } \\
\text { HR }\end{array}$} \\
\hline & $\begin{array}{c}\text { Associated Allele, } \\
\text { HR, 95\% CI }\end{array}$ & $\mathrm{p}$ & \\
\hline \multicolumn{4}{|l|}{ Folate pathway } \\
\hline MTHFR rs 1801133 & $\mathrm{~T}, 1.78,1-3.15$ & 0.050 & 1.41 \\
\hline MTHFR rs 1801131 & $\mathrm{C}, 0.58,0.34-0.98$ & 0.043 & 1 \\
\hline$M T R$ rs 1805087 & $\mathrm{G}, 0.88,0.47-1.65$ & 0.693 & 1 \\
\hline$M T R R$ rs1801394 & $\mathrm{G}, 1.64,0.65-4.13$ & 0.296 & 1.17 \\
\hline \multicolumn{4}{|l|}{ Adenosine pathway } \\
\hline ATIC rs 2372536 & $\mathrm{G}, 0.85,0.5-1.46$ & 0.562 & 1 \\
\hline ITPA rs 1127354 & $\mathrm{~A}, 0.92,0.37-2.33$ & 0.866 & 1 \\
\hline \multicolumn{4}{|l|}{ MTX transporters } \\
\hline$A B C C 2$ rs 2804402 & $\mathrm{G}, 1.07,0.55-2.08$ & 0.84 & 1 \\
\hline$A B C C 2$ rs 2273697 & A, $1.59,0.94-2.68$ & 0.086 & 1.05 \\
\hline
\end{tabular}

Significant data are in bold face. AE: adverse events; SNP: single-nucleotide polymorphism; MTX: methotrexate.

porter SLCO1B1 rs11045879 ( $\mathrm{p}=0.045)$. The association of AMPDI rs17602729 and MTHFD1 rs2236225 remained significant in the multivariable model (HR 1.61 and 1.55, respectively).

In the univariate analysis, no SNP were significantly associated with time to moderately elevated liver transaminase; nevertheless 11 SNP were statistically significantly associated in the multivariate analyses, including MTRR rs1801394 with the strongest association (HR 2.76), MTHFD1 rs2236225 (HR 1.64 for wild-type), MTHFR rs1801133 (HR 1.12), MTR rs1805087 (HR 1.25 for wild-type) TYMS rs34743033 (HR 1.01), ATIC rs2372536 (HR 1.24), ABCB1 rs 1045642 (HR 1.28), ABCG2 rs2231137 (HR 1.54 for wild-type), SLC19A1 rs1051266 (HR 1.10), $A B C C 2$ rs717620 (HR 1.29), and $A B C C 2$ rs2804402 (HR 1.05).

All patients who discontinued MTX because of toxicity had at least 1 polymorphic allele of MTHFR rs1801131 in comparison with $52 \%$ of the patients who tolerated the drug throughout the followup period. None of the patients who discontinued MTX carried any polymorphic alleles of $M T R$ rs $1805087, A B C G 2$ rs 2231137 , and $A B C C 2$ rs 2273697 in comparison with $32 \%, 71 \%$, and $41 \%$ of patients who did not (Table 6).

In the final part of our analyses, we investigated the association between the SNP and the time to escalation to biologic therapy, either because of inefficacy or because of AE. In the univariate analyses, MTHFR rs 1801133 $(\mathrm{p}=0.050)$ and MTHFR rs1801131 $(\mathrm{p}=0.043)$ were significantly associated and in the multivariate analysis, the association of MTHFR rs1801133 (HR 1.41) also persisted with MTRR rs 1801394 (HR 1.17) from folate pathway and transporter $A B C C 2$ rs2273697 (HR 1.05) being associated with escalation to biologic therapy (Table 4).

Table 5. Pharmacogenetic determinants of MTX toxicity. Results of all performed analyses are shown in Supplementary Table 4 (available with the online version of this article).

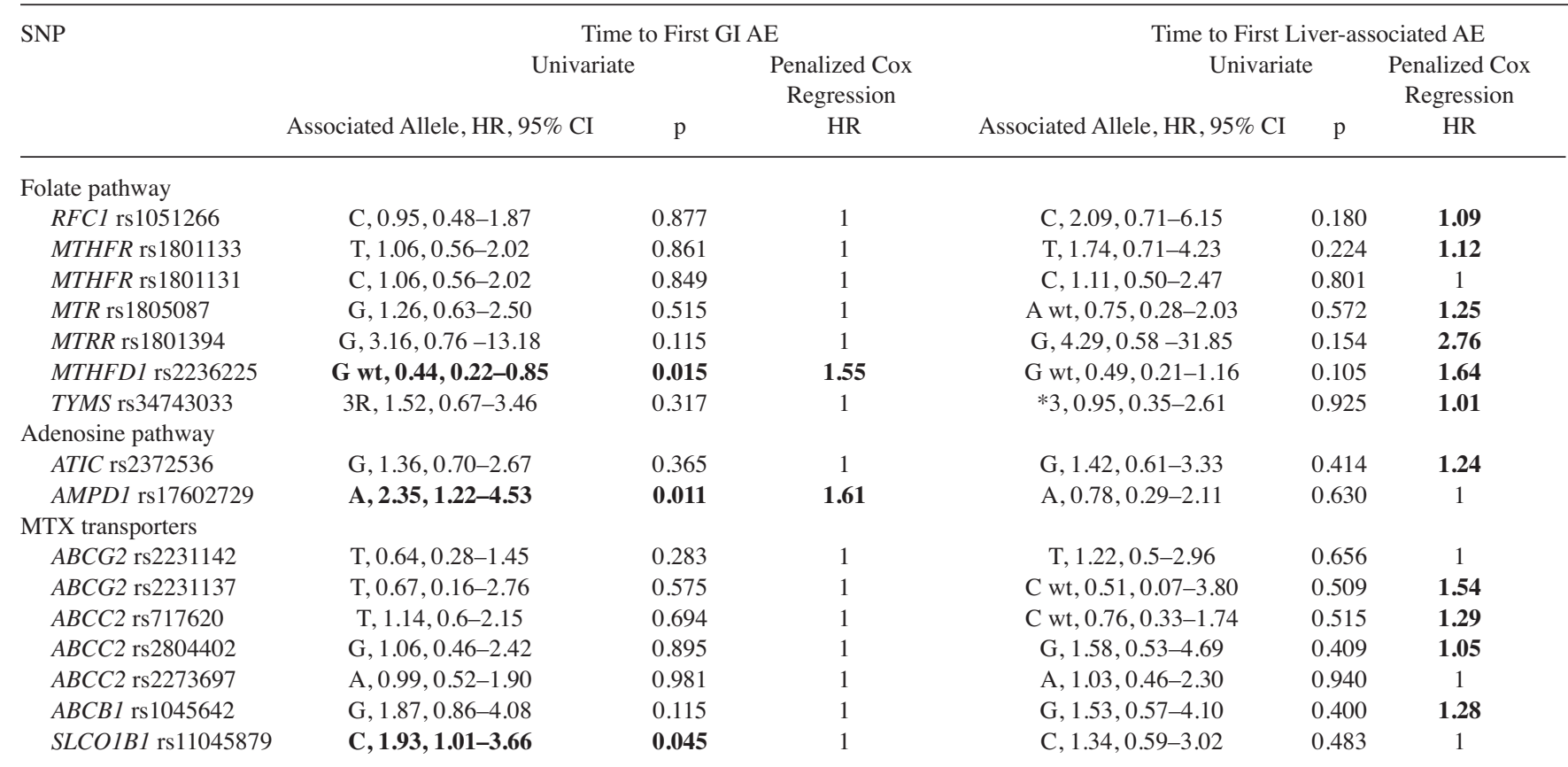

\footnotetext{
* 3 means 3R, triple repeat of 28 base pairs. Wt was associated with tested variable, and in all other analyses, polymorphic allele was associated with tested
} variable. Significant data are in bold face. MTX: methotrexate; GI: gastrointestinal; AE: adverse events; SNP: single-nucleotide polymorphism; wt: wild-type. 
Table 6. Pharmacogenetic determinants of MTX discontinuation because of AE. Percentage of patients carrying at least 1 polymorphic allele is shown. Because of the small number of patients $(n=7)$, statistical analyses were unreliable. Results of all performed analyses are shown in Supplementary Table 4 (available with the online version of this article).

\begin{tabular}{|c|c|c|}
\hline SNP, Allele & $\begin{array}{l}\text { Patients Who Did Not } \\
\text { Discontinue MTX } \\
\text { Because of AE, } \\
n=110\end{array}$ & $\begin{array}{c}\text { Patients Who } \\
\text { Discontinued } \\
\text { MTX Because of } \\
\text { AE, } \mathrm{n}=7\end{array}$ \\
\hline \multicolumn{3}{|l|}{ Folate pathway } \\
\hline RFC1 rs1051266, C & 72 & 43 \\
\hline MTHFR $\mathrm{rs} 1801133, \mathrm{~T}$ & 61 & 29 \\
\hline$M T H F R$ rs $1801131, \mathrm{C}$ & 53 & 100 \\
\hline$M T R \operatorname{rs} 1805087, \mathrm{G}$ & 32 & $\mathbf{0}$ \\
\hline$M T R R$ rs $1801394, \mathrm{G}$ & 86 & 100 \\
\hline MTHFD1 rs2236225, A & 68 & 71 \\
\hline TYMS rs34743033, 3R & 77 & 57 \\
\hline \multicolumn{3}{|l|}{ Adenosine pathway } \\
\hline ATIC rs2372536, G & 59 & 71 \\
\hline AMPD1 rs17602729, A & 25 & 14 \\
\hline \multicolumn{3}{|l|}{ MTX transporters } \\
\hline$A B C G 2$ rs $2231142, \mathrm{~T}$ & 23 & 14 \\
\hline$A B C G 2$ rs $2231137, \mathrm{~T}$ & 71 & $\mathbf{0}$ \\
\hline$A B C C 2$ rs $717620, \mathrm{~T}$ & 41 & 29 \\
\hline$A B C C 2$ rs $2804402, \mathrm{G}$ & 82 & 86 \\
\hline$A B C C 2$ rs $2273697, \mathrm{~A}$ & 41 & $\mathbf{0}$ \\
\hline$A B C B 1 \mathrm{rs} 1045642, \mathrm{G}$ & 72 & 71 \\
\hline SLCO1B1 rs11045879, C & 37 & 57 \\
\hline
\end{tabular}

Significant data are in bold face. MTX: methotrexate; AE: adverse events; SNP: single-nucleotide polymorphism.

\section{DISCUSSION}

The ultimate goal of treating JIA is achieving and sustaining disease remission. MTX is an important drug for the treatment of JIA, but early predictors are needed to identify patients who will not respond to MTX or will develop $\mathrm{AE}^{2}$. In our present study, none of the clinical variables was associated with response to treatment; however, specific genetic markers were identified that may be associated to efficacy and toxicity of MTX in JIA. Our study was based on a well-described patient cohort representative of the whole JIA population followed in the tertiary care pediatric rheumatology center, and was one of the most comprehensive to evaluate common functional SNP in all MTX pathways. Moreover, the longitudinal followup approach allowed us to collect data on AE for a total of 405 patient-years, contributing to the reliability of our findings.

MTX treatment efficacy results of our study are consistent with previous studies demonstrating that almost half of the patients required escalation of therapy to biologics ${ }^{27}$. These represent the most important target group for a therapeutic intervention that could be based on pharmacogenetic data. Different definitions of response to treatment make the comparisons of published studies difficult. Therefore, the JADAS scoring system, which could become the cornerstone for response evaluation because of its completeness and practical application, was used in our study $^{21}$.

AE are the reason for MTX withdrawal in around $20 \%$ of adult patients with rheumatoid arthritis (RA). In children, AE are less pronounced and in our cohort, 10 patients $(8.4 \%)$ discontinued MTX because of AE, a comparable number to other studies ${ }^{8,10}$. Possibly children use less concomitant drugs and are more compliant to folic acid supplementation, which reduces hepatic and $\mathrm{GAE}^{28}$. GAE lower the quality of life and lead to reduced compliance ${ }^{29}$, and these occurred in almost one-third of our patients, which is comparable to previous studies 10,30

Several SNP were evaluated for the first time in JIA in our study. In particular, ABC transporters were evaluated in only a few studies, but have consistently shown statistically significant associations with response. In a study of 285 children with JIA, 2 SNP in ABC transporters were predictors of first-year response to $\mathrm{MTX}^{9}$. These results were not replicated in our cohort; however, according to our clinical experience it seems more appropriate to evaluate MTX effects after 6 months of therapy. In our study, $A B C C 2$ rs2804402, which that has not been analyzed before in JIA, was statistically significantly associated with nonresponse in univariate analysis. ABCC2 rs2273697 was statistically significantly associated with time to inactive disease as well as being involved in switching to biologics. This SNP was evaluated for the first time regarding MTX treatment, emphasizing the need for further pharmacogenetic studies to analyze the involvement of transporters. Nevertheless, a gene within the same subfamily, $A B C C 7$, showed association with response to MTX in a genome-wide association study on 759 patients with $\mathrm{JIA}^{17}$.

In the previous pharmacogenetic studies in patients with JIA, AE were not assessed and not subdivided according to the organ system or severity $8,11,12,31,32$, or only a limited number of SNP were analyzed ${ }^{13,14,33}$. Identifying patients with AE that make them discontinue MTX would be of major clinical importance. Because of a small number of patients experiencing this event in our study $(n=7)$, statistical analyses were unreliable. Interestingly, their genetic background was different, because all these patients carried polymorphic alleles of MTHFR rs1801131 and only wild-type alleles of MTR rs1805087, ABCG2 rs2231137, and $A B C C 2$ rs2273697. MTHFR is a key enzyme in the folate homeostasis. SNP in its gene are widely researched and believed to contribute to reduced enzymatic activity. The role of MTHFR rs1801131 is not consistent; in a study on RA, it was associated with toxicity ${ }^{34}$, but some studies found different or no association $5,10,13,32$. To our knowledge, $A B C C 2$ rs2273697 and rs2804402 have not yet been evaluated in relation to MTX treatment. We hypothesize that the efflux rate of MTX polyglutamates might be decreased in the presence of SNP in ABC transporter genes, resulting in intracellular accumulation of active metabolites ${ }^{18}$.

Personal non-commercial use only. The Journal of Rheumatology Copyright @ 2017 . All rights reserved. 
Gastrointestinal- and liver-associated AE were 2 main reasons to discontinue MTX and both were associated with specific genetic markers. The carriers of AMPDI rs17602729 and of wild-type MTHFD1 rs2236225 had almost $2 \times$ the higher hazard rates for developing GAE compared with mutated patients, which has not been shown in previous studies that focused on assessment of efficacy rather than $\mathrm{AE}^{8,12,35,36}$. In a prediction model of GAE of MTX in JIA, the involvement of AMPDI rs17602729 was not significant $^{11}$. Its functional role is not clear, although it leads to severely truncated peptide ${ }^{3}$. Similarly, MTHFD1 rs223622 is also believed to be a functional SNP affecting enzyme thermostability and shifting the balance of folate pools ${ }^{3,4}$, and in our present study the wild-type also had a 1.64 times higher hazard rate for hepatotoxicity compared with mutated patients. Altogether, 11 SNP were significantly associated with hepatotoxicity and some of them were also associated with treatment discontinuation because of $\mathrm{AE}$, emphasizing that more attention should be paid to $\mathrm{AE}$ in pharmacogenetic studies in children with JIA. Carriers of MTRR rs1801394 had around a $2.5 \times$ higher hazard rate both to develop hepatotoxicity and to discontinue MTX because of AE. Our results are consistent with research in JIA, where MTRR rs1801394 was included in the prediction model for MTX-associated $\mathrm{GAE}^{11}$. Its involvement regarding $\mathrm{AE}$ has been shown in previous studies on $\mathrm{RA}^{5,34}$. Increased risk of toxicity can be explained by low homocysteine remethylation resulting from lower enzymatic activity ${ }^{3}$.

To find pharmacogenetic tests that would be most useful in clinical practice, we have focused on patients who required escalation to biologics, combining inefficacy and AE. To our knowledge, this approach has not been used before. Significant associations were found with MTHFR rs1801133, MTRR rs 1801394, and ABCC2 rs2273697. MTHFR rs1801133 has shown associations with $\mathrm{AE}$ in studies on children as well as in several metaanalyses in patients with $\mathrm{RA}^{13,33,37,38}$. Additionally, it has also been associated with nonresponse to MTX $^{34,39}$. Similarly, MTRR rs1801394 was included in both a prediction model for nonresponse in $\mathrm{JIA}^{9}$ as well as exhibited associations with $\mathrm{AE}$ in our study and previous ones ${ }^{5,34}$. Therefore, it seems that the combined approach used in our study could integrate the different influences of these SNP.

Some limitations of our study should be considered. The size of our cohort was relatively small $(n=119)$ and did not include an independent validation cohort. Patients were followed by the standard protocol used in our department; however, some heterogeneity of our cohort must be taken into account because some patients were reviewed retrospectively. This could also influence the reporting of AE, especially those that are not an objective laboratory result.

Our results suggest that SNP in different MTX metabolic pathways influence treatment with MTX and that genetic variability is a better marker for toxicity than efficacy.
AMPD1 rs17602729 and MTHFD1 rs223622 were associated with GAE while the latter together with MTRR rs 1801394 also demonstrated the strongest association with developing hepatoxicity. MTHFR rs 1801131, MTR rs $1805087, A B C G 2$ rs2231137, and $A B C C 2$ rs2273697 were identified as potential markers for discontinuing MTX treatment because of AE. MTHFR rs1801133, MTRR rs1801394, and ABCC2 rs2273697 were associated with switching to biologics because of inefficacy or toxicity, indicating patients who could benefit most from pharmacogenetic testing in clinical environment. Construction of a prediction model based on both clinical and pharmacogenetic determinants is needed.

\section{ACKNOWLEDGMENT}

We thank Maruša Debeljak who helped with DNA isolation.

\section{ONLINE SUPPLEMENT}

Supplementary material accompanies the online version of this article.

\section{REFERENCES}

1. Beukelman T, Patkar NM, Saag KG, Tolleson-Rinehart S, Cron RQ, DeWitt EM, et al. 2011 American College of Rheumatology recommendations for the treatment of juvenile idiopathic arthritis: initiation and safety monitoring of therapeutic agents for the treatment of arthritis and systemic features. Arthritis Care Res 2011;63:465-82

2. Ravelli A, Martini A. Juvenile idiopathic arthritis [review]. Lancet 2007;369:767-78.

3. Gervasini G. Polymorphisms in methotrexate pathways: what is clinically relevant, what is not, and what is promising. Curr Drug Metab 2009; 10:547-66.

4. Braun J, Rau R. An update on methotrexate. Curr Opin Rheumatol 2009;21:216-23.

5. Bohanec Grabar P, Logar D, Lestan B, Dolzan V. Genetic determinants of methotrexate toxicity in rheumatoid arthritis patients: a study of polymorphisms affecting methotrexate transport and folate metabolism. Eur J Clin Pharmacol 2008;64:1057-68.

6. Grabar PB, Rojko S, Logar D, Dolzan V. Genetic determinants of methotrexate treatment in rheumatoid arthritis patients: a study of polymorphisms in the adenosine pathway. Ann Rheum Dis 2010;69:931-2

7. Malik F, Ranganathan P. Methotrexate pharmacogenetics in rheumatoid arthritis: a status report. Pharmacogenomics 2013;14:305-14

8. Bulatovic M, Heijstek MW, Van Dijkhuizen EH, Wulffraat NM, Pluijm SM, de Jonge R. Prediction of clinical non-response to methotrexate treatment in juvenile idiopathic arthritis. Ann Rheum Dis 2012;71:1484-9.

9. de Rotte MC, Bulatovic M, Heijstek MW, Jansen G, Heil SG, van Schaik RH, et al. ABCB1 and ABCC3 gene polymorphisms are associated with first-year response to methotrexate in juvenile idiopathic arthritis. J Rheumatol 2012;39:2032-40.

10. van Dijkhuizen EP, Wulffraat NM. Prediction of methotrexate efficacy and adverse events in patients with juvenile idiopathic arthritis: a systematic literature review. Pediatr Rheumatol Online J 2014;12:51.

11. van Dijkhuizen EH, Bulatović Ćalasan M, Pluijm SM, de Rotte MC, Vastert SJ, Kamphuis S, et al. Prediction of methotrexate intolerance in juvenile idiopathic arthritis: a prospective, observational cohort study. Pediatr Rheumatol Online J 2015;13:5.

12. Albers HM, Wessels JA, van der Straaten RJ, Brinkman DM, Suijlekom-Smit LW, Kamphuis SS, et al. Time to treatment as an

Personal non-commercial use only. The Journal of Rheumatology Copyright (C) 2017. All rights reserved. 
important factor for the response to methotrexate in juvenile idiopathic arthritis. Arthritis Rheum 2009;61:46-51.

13. Tuková J, Chládek J, Hroch M, Nemcová D, Hoza J, Dolezalová P. 677TT genotype is associated with elevated risk of methotrexate (MTX) toxicity in juvenile idiopathic arthritis: treatment outcome, erythrocyte concentrations of MTX and folates, and MTHFR polymorphisms. J Rheumatol 2010;37:2180-6.

14. Yanagimachi M, Naruto T, Hara T, Kikuchi M, Hara R, Miyamae T, et al. Influence of polymorphisms within the methotrexate pathway genes on the toxicity and efficacy of methotrexate in patients with juvenile idiopathic arthritis. Br J Clin Pharmacol 2011;71:237-43.

15. Schmeling H, Horneff G, Benseler SM, Fritzler MJ. Pharmacogenetics: can genes determine treatment efficacy and safety in JIA? Nat Rev Rheumatol 2014;10:682-90.

16. Pastore S, Stocco G, Moressa V, Zandonà L, Favretto D, Malusà N, et al. 5-Aminoimidazole-4-carboxamide ribonucleotide-transformylase and inosine-triphosphate-pyrophosphatase genes variants predict remission rate during methotrexate therapy in patients with juvenile idiopathic arthritis. Rheumatol Int 2015;35:619-27.

17. Cobb J, Cule E, Moncrieffe H, Hinks A, Ursu S, Patrick F, et al. Genome-wide data reveal novel genes for methotrexate response in a large cohort of juvenile idiopathic arthritis cases. Pharmacogenomics J 2014;14:356-64.

18. Franke RM, Gardner ER, Sparreboom A. Pharmacogenetics of drug transporters. Curr Pharm Des 2010;16:220-30.

19. Lopez-Lopez E, Martin-Guerrero I, Ballesteros J, Piñan MA, Garcia-Miguel P, Navajas A, et al. Polymorphisms of the SLCO1B1 gene predict methotrexate-related toxicity in childhood acute lymphoblastic leukemia. Pediatr Blood Cancer 2011;57:612-9.

20. Petty RE, Southwood TR, Manners P, Baum J, Glass DN, Goldenberg J, et al; International League of Associations for Rheumatology. International League of Associations for Rheumatology classification of juvenile idiopathic arthritis: second revision, Edmonton, 2001. J Rheumatol 2004;31:390-2.

21. Consolaro A, Ruperto N, Bazso A, Pistorio A, Magni-Manzoni S, Filocamo G, et al; Paediatric Rheumatology International Trials Organisation. Development and validation of a composite disease activity score for juvenile idiopathic arthritis. Arthritis Rheum 2009;61:658-66.

22. Consolaro A, Bracciolini G, Ruperto N, Pistorio A, Magni-Manzoni S, Malattia C, et al; Paediatric Rheumatology International Trials Organization. Remission, minimal disease activity, and acceptable symptom state in juvenile idiopathic arthritis: defining criteria based on the juvenile arthritis disease activity score. Arthritis Rheum 2012;64:2366-74.

23. Wallace CA, Ravelli A, Huang B, Giannini EH. Preliminary validation of clinical remission criteria using the OMERACT filter for select categories of juvenile idiopathic arthritis. J Rheumatol 2006;33:789-95.

24. U.S. Department of Health and Human Services. Common terminology criteria for adverse events (CTCAE) version 4.03. [Internet. Accessed April 10, 2017.] Available from: evs.nci.nih.gov/ftp1/CTCAE/CTCAE_4.03_2010-06-14_ QuickReference_5x7.pdf

25. Iacopetta B, Grieu F, Joseph D, Elsaleh H. A polymorphism in the enhancer region of the thymidylate synthase promoter influences the survival of colorectal cancer patients treated with 5-fluorouracil. $\mathrm{Br}$ J Cancer 2001;85:827-30.
26. Team RC. R: a language and environment for statistical computing. Vienna: R Foundation for Statistical Computing; 2015.

27. Maetzel A, Wong A, Strand V, Tugwell P, Wells G, Bombardier C. Meta-analysis of treatment termination rates among rheumatoid arthritis patients receiving disease-modifying anti-rheumatic drugs. Rheumatology 2000;39:975-81.

28. Prey S, Paul C. Effect of folic or folinic acid supplementation on methotrexate-associated safety and efficacy in inflammatory disease: a systematic review. Br J Dermatol 2009;160:622-8.

29. Brunner HI, Johnson AL, Barron AC, Passo MH, Griffin TA, Graham TB, et al. Gastrointestinal symptoms and their association with health-related quality of life of children with juvenile rheumatoid arthritis: validation of a gastrointestinal symptom questionnaire. J Clin Rheumatol 2005;11:194-204.

30. Bulatović M, Heijstek MW, Verkaaik M, van Dijkhuizen EH, Armbrust W, Hoppenreijs EP, et al. High prevalence of methotrexate intolerance in juvenile idiopathic arthritis: development and validation of a methotrexate intolerance severity score. Arthritis Rheum 2011;63:2007-13.

31. Hinks A, Moncrieffe H, Martin P, Ursu S, Lal S, Kassoumeri L, et al. Association of the 5-aminoimidazole-4-carboxamide ribonucleotide transformylase gene with response to methotrexate in juvenile idiopathic arthritis. Ann Rheum Dis 2011;70:1395-400.

32. Scheuern A, Fischer N, McDonald J, Brunner HI, Haas JP, Hügle B. Mutations in the MTHFR gene are not associated with Methotrexate intolerance in patients with juvenile idiopathic arthritis. Pediatr Rheumatol 2016;14:11.

33. Schmeling H, Biber D, Heins S, Horneff G. Influence of methylenetetrahydrofolate reductase polymorphisms on efficacy and toxicity of methotrexate in patients with juvenile idiopathic arthritis. J Rheumatol 2005;32:1832-6.

34. Dervieux T, Greenstein N, Kremer J. Pharmacogenomic and metabolic biomarkers in the folate pathway and their association with methotrexate effects during dosage escalation in rheumatoid arthritis. Arthritis Rheum 2006;54:3095-103.

35. Hinks A, Barton A, Shephard N, Eyre S, Bowes J, Cargill M, et al; British Society of Paediatric and Adolescent Rheumatology Study Group. Identification of a novel susceptibility locus for juvenile idiopathic arthritis by genome-wide association analysis. Arthritis Rheum 2009;60:258-63.

36. Wessels JA, van der Kooij SM, le Cessie S, Kievit W, Barerra P, Allaart CF, Huizinga TW, et al; Pharmacogenetics Collaborative Research Group. A clinical pharmacogenetic model to predict the efficacy of methotrexate monotherapy in recent-onset rheumatoid arthritis. Arthritis Rheum 2007;56:1765-75.

37. Fisher MC, Cronstein BN. Metaanalysis of methylenetetrahydrofolate reductase (MTHFR) polymorphisms affecting methotrexate toxicity. J Rheumatol 2009;36:539-45.

38. Song GG, Bae SC, Lee YH. Association of the MTHFR C677T and A1298C polymorphisms with methotrexate toxicity in rheumatoid arthritis: a meta-analysis. Clin Rheumatol 2014;33:1715-24.

39. Campalani E, Arenas M, Marinaki AM, Lewis CM, Barker JN, Smith CH. Polymorphisms in folate, pyrimidine, and purine metabolism are associated with efficacy and toxicity of methotrexate in psoriasis. J Invest Dermatol 2007;127:1860-7. 